\title{
Handling Constraints in Particle Swarm Optimization using a Small Population Size
}

\author{
Juan C. Fuentes Cabrera and Carlos A. Coello Coello \\ CINVESTAV-IPN (Evolutionary Computation Group) \\ Departamento de Computación \\ Av. IPN No. 2508, San Pedro Zacatenco \\ México D.F. 07360, MÉXICO \\ jfuentes@computacion.cs.cinvestav.mx \\ ccoello@cs.cinvestav.mx
}

\begin{abstract}
This paper presents a particle swarm optimizer for solving constrained optimization problems which adopts a very small population size (five particles). The proposed approach uses a reinitialization process for preserving diversity, and does not use a penalty function nor it requires feasible solutions in the initial population. The leader selection scheme adopted is based on the distance of a solution to the feasible region. In addition, a mutation operator is incorporated to improve the exploratory capabilities of the algorithm. The approach is tested with a well-know benchmark commonly adopted to validate constrainthandling approaches for evolutionary algorithms. The results show that the proposed algorithm is competitive with respect to state-of-the-art constraint-handling techniques. The number of fitness function evaluations that the proposed approach requires is almost the same (or lower) than the number required by the techniques of the state-of-the-art in the area.
\end{abstract}

\section{Introduction}

A wide variety of Evolutionary Algorithms (EAs) have been used to solve different types of optimization problems. However, EAs are unconstrained search techniques and thus require an additional mechanism to incorporate the constraints of a problem into their fitness function [3]. Penalty functions are the most commonly adopted approach to incorporate constraints into an EA. However, penalty functions have several drawbacks, from which the most important are that they require a fine-tuning of the penalty factors (which are problemdependent), since both under- and over-penalizations may result in an unsuccessful optimization [3].

Particle swarm optimization (PSO) is a population based optimization technique inspired on the movements of a flock of birds or fish. PSO has been successfully applied in a wide of variety of optimization tasks in which it has shown a high convergence rate [10].

This paper is organized as follows. In section 2, we define the problem of our interest and we introduce the Particle Swarm Optimization algorithm. The 
previous related work is provided in Section 3. Section 4 describes our approach including the reinitialization process, the constraint-handling mechanism and the mutation operator adopted. In Section 5, we present our experimental setup and the results obtained. Finally, Section 6 presents our conclusions and some possible paths for future research.

\section{Basic Concepts}

We are interested in the general nonlinear programming problem in which we want to:

$$
\text { Find } \vec{x} \text { which optimizes } f(\vec{x})
$$

subject to:

$$
\begin{aligned}
g_{i}(\vec{x}) & \leq 0, i=1, \ldots, n \\
h_{j}(\vec{x}) & =0, j=1, \ldots, p
\end{aligned}
$$

where $\vec{x}$ is the vector of solutions $\vec{x}=\left[x_{1}, x_{2}, \ldots, x_{r}\right]^{T}, n$ is the number of inequality constraints and $p$ is the number of equality constraints (in both cases, constraints could be linear or nonlinear). If we denote with $F$ to the feasible region (set of points which satisfy the inequality and equality constraints) and with $S$ to the search space then $F \subseteq S$. For an inequality constraint that satisfies $g_{i}(\boldsymbol{x})=0$, the we will say that is active at $\vec{x}$. All equality constraints $h_{j}$ (regardless of the value of $\vec{x}$ used) are considered active at all points of $F$.

\subsection{Particle Swarm Optimization}

Our approach is based in The Particle Swarm Optimization (PSO) algorithm which was introduced by Eberhart and Kennedy in 1995 [4]. PSO is a populationbased search algorithm based on the simulation of social behavior of birds within a flock [10]. In PSO, each individual (particle) of the population (swarm) adjusts its trajectory according to its own flying experience and the flying experience of the other particles within its topological neighborhood in the search space. In the PSO algorithm, the population and velocities are randomly initialized at the beginning of the search, and then they are iteratively updated, based on their previous positions and those of each particle's neighbors. Our proposed approach implements equations (4) and (5), proposed in [19] for computing the velocity and the position of a particle.

$$
\begin{gathered}
v_{i d}=w \times v_{i d}+c_{1} r_{1}\left(p b_{i d}-x_{i d}\right)+c_{2} r_{2}\left(l b_{i d}-x_{i d}\right) \\
x_{i d}=x_{i d}+v_{i d}
\end{gathered}
$$

where $c_{1}$ and $c_{2}$ are both positive constants, $r_{1}$ and $r_{2}$ are random numbers generated from a uniform distribution in the range $[0,1], w$ is the inertia weight that is generated in the range $(0,1]$. 
There are two versions of the PSO algorithm: the global version and the local version. In the global version, the neighborhood consists of all the particles of the swarm and the best particle of the population is called the "global best" (gbest). In contrast, in the local version, the neighborhood is a subset of the population and the best particle of the neighborhood is called "local best" (lbest).

\section{Related Work}

When incorporating constraints into the fitness function of an evolutionary algorithm, it is particularly important to maintain diversity in the population and to be able to keep solutions both inside and outside the feasible region $[3,14]$. Several studies have shown that, despite their popularity, traditional (external) penalty functions, even when used with dynamic penalty factors, tend to have difficulties to deal with highly constrained search spaces and with problems in which the constraints are active in the optimum [3,11,18]. Motivated by this fact, a number of constraint-handling techniques have been proposed for evolutionary algorithms $[16,3]$.

Remarkably, there has been relatively little work related to the incorporation of constraints into the PSO algorithm, despite the fact that most real-world applications have constraints. In previous work, some researchers have assumed the possibility of being able to generate in a random way feasible solutions to feed the population of a PSO algorithm $[7,8]$. The problem with this approach is that it may have a very high computational cost in some cases. For example, in some of the test functions used in this paper, even the generation of one million of random points was insufficient to produce a single feasible solution. Evidently, such a high computational cost turns out to be prohibitive in realworld applications.

Other approaches are applicable only to certain types of constraints (see for example [17]). Some of them rely on relatively simple mechanisms to select a leader, based on the closeness of a particle to the feasible region, and adopt a mutation operator in order to maintain diversity during the search (see for example [20]). Other approaches rely on carefully designed topologies and diversity mechanisms (see for example [6]). However, a lot of work remains to be done regarding the use of PSO for solving constrained optimization problems. For example, the use of very small population sizes has not been addressed so far in PSO, to the authors' best knowledge, and this paper precisely aims to explore this venue in the context of constrained optimization.

\section{Our Proposed Approach}

As indicated before, our proposed approach is based on the local version of PSO, since there is evidence that such model is less prone to getting stuck in local minima [10]. Despite the existence of a variety of population topologies [9], we adopt a randomly generated neighborhood topology for our approach. It also uses a reinitialization process in order to maintain diversity in the population, and it 
adopts a mutation operator that aims to improve the exploratory capabilities of PSO (see Algorithm 1).

Since our proposed approach uses a very small population size, we called it Micro-PSO, since this is analogous to the micro genetic algorithms that have been in use for several years (see for example $[12,2]$ ). Its constraint-handling mechanism, together with its reinitialization process and its mutation operator, are all described next in more detail.

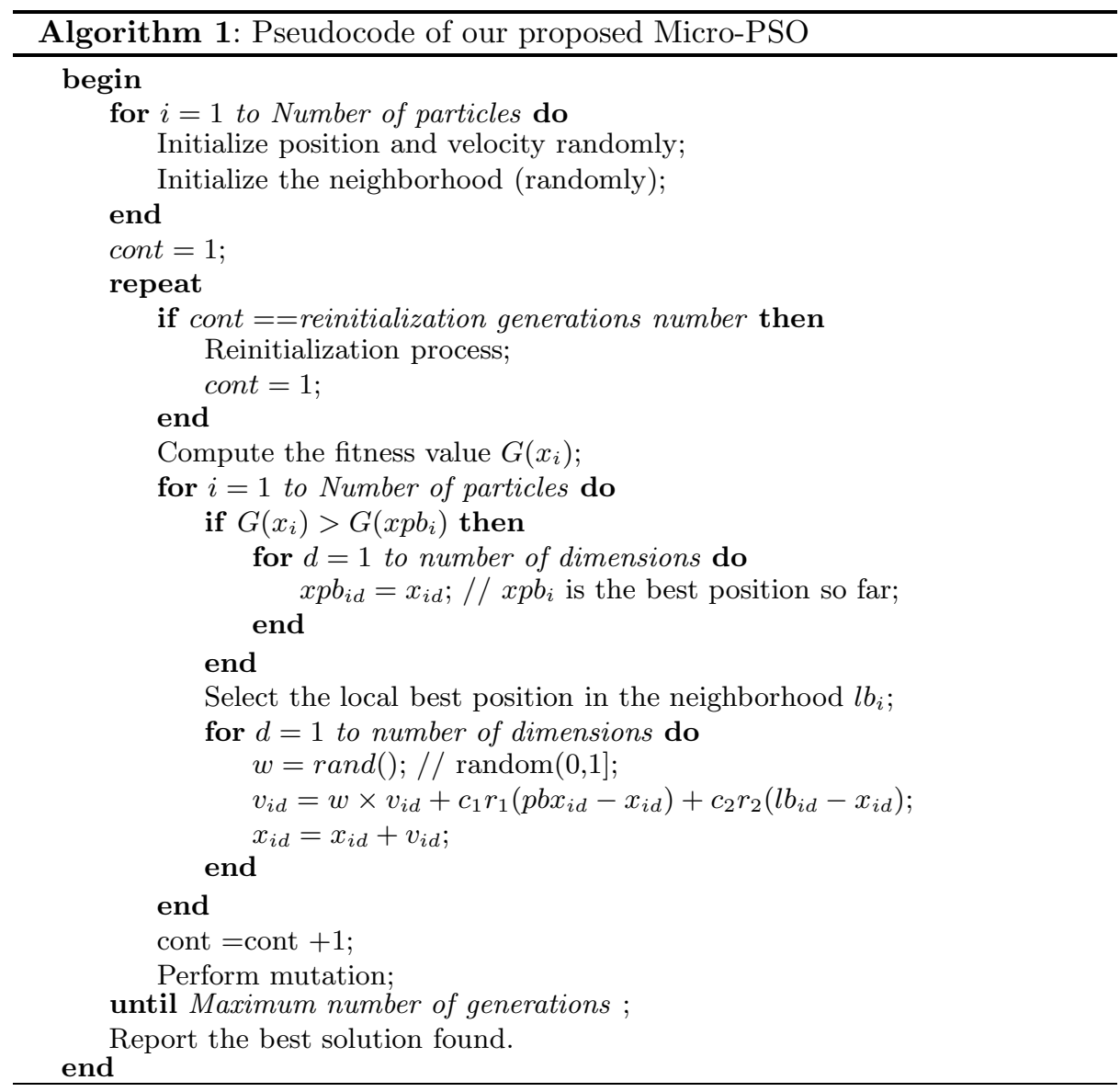

\subsection{Constraint-Handling Mechanism}

We adopted the mechanism proposed in [20] for selecting leaders. This mechanism is based both on the feasible solutions and the fitness value of a particle. When two feasible particles are compared, the particle that has the highest fitness value wins. If one of the particles is infeasible and the other one is feasible, 
then the feasible particle wins. When two infeasible particles are compared, the particle that has the lowest fitness value wins. The idea is to select leaders that, even when could be infeasible, lie close to the feasible region.

We used equation (6) for assigning fitness to a solution:

$$
f i t(\vec{x})=\left\{\begin{array}{cl}
f_{i}(\vec{x}) & \text { if feasible } \\
\sum_{j=1}^{n} g_{j}(\vec{x})+\sum_{k=1}^{p}\left|h_{k}(\vec{x})\right| & \text { otherwise }
\end{array}\right.
$$

\subsection{Reinitialization Process}

The use of a small population size accelerates the loss of diversity at each iteration, and therefore, it is uncommon practice to use population sizes that are too small. However, in the genetic algorithms literature, it is known that it is possible, from a theoretical point of view, to use very small population sizes (no more than 5 individuals) if appropriate reinitialization processes are implemented [12, 2]. In this paper, we incorporate one of these reinitialization processes taken from the literature on micro genetic algorithms. Our mechanism is the following: after certain number of iterations (replacement generations), the swarm is sorted based on fitness value, but placing the feasible solutions on top. Then, we replace the $r p$ particles (replacement particles) by randomly generated particles (position and velocity), but allow the rp particles to hold their best position (pbest). The idea of mixing evolved and randomly generated particles is to avoid premature convergence.

\subsection{Mutation Operator}

Although the original PSO algorithm had no mutation operator, the addition of such mutation operator is a relatively common practice nowadays. The main motivation for adding this operator is to improve the performance of PSO as an optimizer, and to improve the overall exploratory capabilities of this heuristic [1]. In our proposed approach, we implemented the mutation operator developed by Michalewicz for Genetic Algorithms [15]. It is worth noticing that this mutation operator has been used before in PSO, but in the context of unconstrained multimodal optimization [5]. This operator varies the magnitude added or substracted to a solution during the actual mutation, depending on the current iteration number (at the beginning of the search, large changes are allowed, and they become very small towards the end of the search). We apply the mutation operator in the particle's position, for all of its dimensions:

$$
x_{i d}= \begin{cases}x_{i d}+\Delta\left(t, U B-x_{i d}\right) & \text { if } R=0 \\ x_{i d}-\Delta\left(t, x_{i d}-L B\right) & \text { if } R=1\end{cases}
$$

where $t$ is the current iteration number, $U B$ is the upper bound on the value of the particle dimension, $L B$ is the lower bound on the particle dimension value, $R$ is a randomly generated bit (zero and one both have a $50 \%$ probability of being generated) and $\delta(t, y)$ returns a value in the range $[0, y] . \delta(t, y)$ is defined by: 


$$
\Delta(t, y)=y *\left(1-r^{1-\left(\frac{t}{T}\right)^{b}}\right)
$$

where $r$ is a random number generated from a uniform distribution in the range $[0,1], T$ is the maximum number of iterations and $b$ is a tunable parameter that defines the non-uniformity level of the operator. In this approach, the $b$ parameter is set to 5 as suggested in [15].

\section{Experiments and Results}

For evaluating the performance of our proposed approach, we used the thirteen test functions described in [18]. These test functions contain characteristics that make them difficult to solve using evolutionary algorithms. We performed fifty independent runs for each test function and we compared our results with respect to three algorithms representative of the state-of-the-art in the area: Stochastic Ranking (SR) [18], the Simple Multimembered Evolution Strategy (SMES) [14], and the Constraint-Handling Mechanism for PSO (CHM-PSO) [20]. Stochastic Ranking uses a multimembered evolution strategy with a static penalty function and a selection based on a stochastic ranking process, in which the stochastic component allows infeasible solutions to be given priority a few times during the selection process. The idea is to balance the influence of the objective and penalty function. This approach requires a user-defined parameter called $P f$ wich determines this balance [18]. The Simple Multimembered Evolution Strategy (SMES) is based on a $(\mu+\lambda)$ evolution strategy, and it has three main mechanisms: a diversity mechanism, a combined recombination operator and a dynamic step size that defines the smoothness of the movements performed by the evolution strategy [14].

In all our experiments, we adopted the following parameters:

- $\mathrm{W}=$ random number from a uniform distribution in the range $[0,1]$.

$-\mathrm{C} 1=\mathrm{C} 2=1.8$.

- population size $=5$ particles;

- number of generations $=48,000$;

- number of replacement generations $=100$;

- number of replacement particles $=2$;

- mutation percent $=0.1$;

$-\epsilon=0.0001$, except for g04, g05, g06, g07, in which we used $\epsilon=0.00001$.

The statistical results of our Micro-PSO are summarized in Table 1. Our approach was able to find the global optimum in five test functions (g02, g04, g06, g08, g09, g12) and it found solutions very close to the global optimum in the remaining test functions, with the exception of g10. We compare the population size, the tolerance value and the number of evaluations of the objective function of our approach with respect to SR, CHM-PSO, and SMES in Table 2.

When comparing our approach with respect to SR, we can see that ours found a better solution for g02 and similar results in other eleven problems, except for 
Table 1. Results obtained by our Micro-PSO over 50 independent runs.

\begin{tabular}{|c|c|c|c|c|c|c|}
\hline \multicolumn{7}{|c|}{ Statistical Results of our Micro-PSO } \\
\hline TF & Optimal & Best & Mean & Median & Worst & St.Dev. \\
\hline g01 & -15.000 & -15.0001 & -13.2734 & -13.0001 & -9.7012 & $1.41 \mathrm{E}+00$ \\
\hline g02 & 0.803619 & 0.803620 & 0.777143 & 0.778481 & 0.711603 & $1.91 \mathrm{E}-02$ \\
\hline g03 & 1.000 & 1.0004 & 0.9936 & 1.0004 & 0.6674 & $4.71 \mathrm{E}-02$ \\
\hline $\mathrm{g} 04$ & -30665.539 & -30665.5398 & -30665.5397 & -30665.5398 & -30665.5338 & $6.83 \mathrm{E}-04$ \\
\hline $\mathrm{g} 05$ & 5126.4981 & 5126.6467 & 5495.2389 & 5261.7675 & 6272.7423 & $4.05 \mathrm{E}+02$ \\
\hline $\mathrm{g} 06$ & -6961.81388 & -6961.8371 & -6961.8370 & -6961.8371 & -6961.8355 & $2.61 \mathrm{E}-04$ \\
\hline $\mathrm{g} 07$ & 24.3062 & 24.3278 & 24.6996 & 24.6455 & 25.2962 & $2.52 \mathrm{E}-01$ \\
\hline $\mathrm{g} 08$ & 0.095825 & 0.095825 & 0.095825 & 0.095825 & 0.095825 & $0.00 \mathrm{E}+00$ \\
\hline $\mathrm{g} 09$ & 680.630 & 680.6307 & 680.6391 & 680.6378 & 680.6671 & $6.68 \mathrm{E}-03$ \\
\hline $\mathrm{g} 10$ & 7049.250 & 7090.4524 & 7747.6298 & 7557.4314 & 10533.6658 & $5.52 \mathrm{E}+02$ \\
\hline $\mathrm{g} 11$ & 0.750 & 0.7499 & 0.7673 & 0.7499 & 0.9925 & $6.00 \mathrm{E}-02$ \\
\hline g12 & 1.000 & 1.0000 & 1.0000 & 1.0000 & 1.0000 & $0.00 \mathrm{E}+00$ \\
\hline g13 & 0.05395 & 0.05941 & 0.81335 & 0.90953 & 2.44415 & $3.81 \mathrm{E}-01$ \\
\hline
\end{tabular}

g10, in which our approach does not perform well (see Table 3). Note however that SR performs 350,000 objective function evaluations and our approach only performs 240,000.

Table 2. Comparison of the population size, the tolerance value and the number of evaluations of the objective function of our approach with respect to SR, CHM-PSO and SMES.

\begin{tabular}{|c|c|c|c|}
\hline $\begin{array}{c}\text { Constraint-Handling } \\
\text { Technique }\end{array}$ & Population Size & Tolerance Value $(\epsilon)$ & $\begin{array}{c}\text { Number of Evaluations } \\
\text { of the Objective Function }\end{array}$ \\
\hline \hline SR & 200 & 0.001 & 350,000 \\
\hline CHMPSO & 40 & - & 340,000 \\
\hline SMES & 100 & 0.0004 & 240,000 \\
\hline Micro-PSO & 5 & 0.0001 & 240,000 \\
\hline
\end{tabular}

Compared with respect to CHM-PSO, our approach found a better solution for g02, g03, g04, g06, g07, g09, and g13 and the same or similar results in other five problems (except for g10) (see Table 4). Note again that CMPSO performs 340,000 objective function evaluations, and our approach performs only 240,000. It is also worth indicating that CHMOPSO is one of the best PSO-based constraint-handling methods known to date.

When comparing our proposed approach against the SMES, ours found better solutions for g02 and g09 and the same or similar results in other ten prob- 
lems, except for g10 (see Table 5). Both approaches performed 240,000 objective function evaluations.

Table 3. Comparison of our approach with respect the Stochastic Ranking (SR).

\begin{tabular}{|l|c|c|c|c|c|c|c|}
\hline & & \multicolumn{2}{|c|}{ Best Result } & \multicolumn{2}{c|}{ Mean Result } & \multicolumn{2}{c|}{ Worst Result } \\
\hline TF & Optimal & Micro-PSO & SR & Micro-PSO & SR & Micro-PSO & SR \\
\hline \hline g01 & -15.0000 & -15.0001 & -15.000 & -13.2734 & -15.000 & -9.7012 & -15.000 \\
\hline g02 & 0.803619 & 0.803620 & 0.803515 & 0.777143 & 0.781975 & 0.711603 & 0.726288 \\
\hline g03 & 1.0000 & 1.0004 & 1.000 & 0.9936 & 1.000 & 0.6674 & 1.000 \\
\hline g04 & -30665.539 & -30665.539 & -30665.539 & -30665.539 & -30665.539 & -30665.534 & -30665.539 \\
\hline g05 & 5126.4981 & 5126.6467 & 5126.497 & 5495.2389 & 5128.881 & 6272.7423 & 5142.472 \\
\hline g06 & -6961.81388 & -6961.8371 & -6961.814 & -6961.8370 & -6875.940 & -6961.8355 & -6350.262 \\
\hline g07 & 24.3062 & 24.3278 & 24.307 & 24.6996 & 24.374 & 25.2962 & 24.642 \\
\hline g08 & 0.095825 & 0.095825 & 0.095825 & 0.095825 & 0.095825 & 0.095825 & 0.095825 \\
\hline g09 & 680.630 & 680.6307 & 680.630 & 680.6391 & 680.656 & 680.6671 & 680.763 \\
\hline g10 & 7049.250 & 7090.4524 & 7054.316 & 7747.6298 & 7559.192 & 10533.6658 & 8835.655 \\
\hline g11 & 0.750 & 0.7499 & 0.750 & 0.7673 & 0.750 & 0.9925 & 0.750 \\
\hline g12 & 1.000 & 1.0000 & 1.000 & 1.0000 & 1.000 & 1.0000 & 1.000 \\
\hline g13 & 0.05395 & 0.05941 & 0.053957 & 0.81335 & 0.067543 & 2.44415 & 0.216915 \\
\hline
\end{tabular}

\section{Conclusions and Future Work}

We have proposed the use of a PSO algorithm with a very small population size (only five particles) for solving constrained optimization problems. The proposed technique adopts a constraint-handling mechanism during the selection of leaders, it performs a reinitialization process and it implements a mutation operator. The proposed approach is easy to implement, since its main additions are a sorting and a reinitialization process. The computational cost (measured in terms of the number of evaluation of the objective function) that our MicroPSO requires is almost the same (or lower) than the number required by the techniques with respect to which it was compared, which are representative of the state-of-the-art in the area. The results obtained show that our approach is competitive and could then, be a viable alternative for using PSO for solving constrained optimization problems.

As part of our future work, we are interested in comparing our approach with the extended benchmark for constrained evolutionary optimization [13]. We are also interested in comparing our results with respect to more recent constrainthandling methods that use PSO as their search engine (see for example [6]). We will also study the sensitivity of our Micro-PSO to its parameters, aiming to find a set of parameters (or a self-adaptation mechanism) that improves its robustness (i.e., that reduces the standard deviations obtained). Finally, we are also interested in experimenting with other neighborhood topologies and other types of 
Table 4. Comparison of our approach with respect the Constraint-Handling Mechanism for PSO (CHM-PSO).

\begin{tabular}{|l|c|c|c|c|c|c|c|}
\hline & & \multicolumn{2}{|c|}{ Best Result } & \multicolumn{2}{c|}{ Mean Result } & \multicolumn{2}{c|}{ Worst Result } \\
\hline TF & Optimal & Micro-PSO & CHM-PSO & Micro-PSO & CHM-PSO & Micro-PSO & CHM-PSO \\
\hline \hline g01 & -15.0000 & -15.0001 & -15.000 & -13.2734 & -15.000 & -9.7012 & -15.000 \\
\hline g02 & 0.803619 & 0.803620 & 0.803432 & 0.777143 & 0.790406 & 0.711603 & 0.755039 \\
\hline g03 & 1.000 & 1.0004 & 1.004720 & 0.9936 & 1.003814 & 0.6674 & 1.000249 \\
\hline g04 & -30665.539 & -30665.539 & -30665.500 & -30665.539 & -30665.500 & -30665.534 & -30665.500 \\
\hline g05 & 5126.4981 & 5126.6467 & 5126.6400 & 5495.2389 & 5461.08133 & 6272.7423 & 6104.7500 \\
\hline g06 & -6961.8138 & -6961.837 & -6961.810 & -6961.837 & -6961.810 & -6961.835 & -6961.810 \\
\hline g07 & 24.3062 & 24.3278 & 24.3511 & 24.6996 & 24.35577 & 25.2962 & 27.3168 \\
\hline g08 & 0.095825 & 0.095825 & 0.095825 & 0.095825 & 0.095825 & 0.095825 & 0.095825 \\
\hline g09 & 680.6300 & 680.6307 & 680.638 & 680.6391 & 680.85239 & 680.6671 & 681.553 \\
\hline g10 & 7049.2500 & 7090.4524 & 7057.5900 & 7747.6298 & 7560.04785 & 10533.6658 & 8104.3100 \\
\hline g11 & 0.7500 & 0.7499 & 0.7499 & 0.7673 & 0.7501 & 0.9925 & 0.75288 \\
\hline g12 & 1.0000 & 1.0000 & 1.000 & 1.0000 & 1.000 & 1.0000 & 1.000 \\
\hline g13 & 0.05395 & 0.05941 & 0.068665 & 0.81335 & 1.71642 & 2.44415 & 13.6695 \\
\hline
\end{tabular}

Table 5. Comparison of our approach with respect the Simple Multimembered Evolution Strategy (SMES).

\begin{tabular}{|l|c|c|c|c|c|c|c|}
\hline & & \multicolumn{2}{|c|}{ Best Result } & \multicolumn{2}{c|}{ Mean Result } & \multicolumn{2}{c|}{ Worst Result } \\
\hline TF & Optimal & Micro-PSO & SMES & Micro-PSO & SMES & Micro-PSO & SMES \\
\hline \hline g01 & -15.0000 & -15.0001 & -15.000 & -13.2734 & -15.000 & -9.7012 & -15.000 \\
\hline g02 & 0.803619 & 0.803620 & 0.803601 & 0.777143 & 0.785238 & 0.711603 & 0.751322 \\
\hline g03 & 1.0000 & 1.0004 & 1.000 & 0.9936 & 1.000 & 0.6674 & 1.000 \\
\hline g04 & -30665.5390 & -30665.5398 & -30665.539 & -30665.5397 & -30665.539 & -30665.5338 & -30665.539 \\
\hline g05 & 5126.4980 & 5126.6467 & 5126.599 & 5495.2389 & 5174.492 & 6272.7423 & 5304.167 \\
\hline g06 & -6961.8140 & -6961.8371 & -6961.814 & -6961.8370 & -6961.284 & -6961.8355 & -6952.482 \\
\hline g07 & 24.3062 & 24.3278 & 24.327 & 24.6996 & 24.475 & 25.2962 & 24.843 \\
\hline g08 & 0.095825 & 0.095825 & 0.095825 & 0.095825 & 0.095825 & 0.095825 & 0.095825 \\
\hline g09 & 680.6300 & 680.6307 & 680.632 & 680.6391 & 680.643 & 680.6671 & 680.719 \\
\hline g10 & 7049.2500 & 7090.4524 & 7051.903 & 7747.6298 & 7253.047 & 10533.6658 & 7638.366 \\
\hline g11 & 0.7500 & 0.7499 & 0.75 & 0.7673 & 0.75 & 0.9925 & 0.75 \\
\hline g12 & 1.0000 & 1.0000 & 1.000 & 1.0000 & 1.000 & 1.0000 & 1.000 \\
\hline g13 & 0.05395 & 0.05941 & 0.053986 & 0.81335 & 0.166385 & 2.44415 & 0.468294 \\
\hline
\end{tabular}


reinitialization processes, since they could improve our algorithm's convergence capabilities (i.e., we could reduce the number of objective function evaluations performed) as well as the quality of the results achieved.

\section{Acknowledgements}

The first author gratefully acknowledges support from CONACyT to pursue graduate studies at CINVESTAV-IPN's Computer Science Department. The second author acknowledges support from CONACyT project No. 45683-Y.

\section{References}

1. Paul S. Andrews. An investigation into mutation operators for particle swarm optimization. In Proceedings of the 2006 IEEE Congress on Evolutionary Computation, (CEC'2006), pages 3789-3796, Vancouver, Canada, July 2006.

2. Carlos A. Coello Coello and Gregorio Toscano Pulido. Multiobjective optimization using a micro-genetic algorithm. In in L. Spector, E.D. Goodman, A. Wu, W. Langdon, H.M. Voigt, M. Gen, S. Sen, M. Dorigo, S. Pezeshk M., Garzon, and E. Burke, editors, Genetic and Evolutionary Computation Conference, GECCO,2001, pages 274-282, San Francisco, California, 2001. Morgan Kaufmann Publishers.

3. Carlos A. Coello Coello. Theoretical and numerical constraint handling techniques used with evolutionary algorithms: A survey of the state of the art. Computer Methods in Applied Mechanics and Engineering, 191(11-12):1245-1287, January 2002.

4. R. Eberhart and J. Kennedy. Particle swarm optimization. In Proceedings of the IEEE International Conference on Neural Networks, pages 1942-1948. IEEE Press, 1995.

5. S.C. Esquivel and C.A. Coello Coello. On the use of particle swarm optimization with multimodal functions. In Proceedings of the 2003 IEEE Congress on Evolutionary Computation (CEC'2003), pages 1130-1136, Canberra, Australia, 2003. IEEE Press.

6. Arturo Hernández Aguirre, Angel E. Muñoz Zavala, E. Villa Diharce, and S. Botello Rionda. COPSO: Constrained Optimization via PSO algorithm. Technical Report I-07-04, Center of Research in Mathematics (CIMAT), Guanajuato, México, 2007.

7. Xiaohui $\mathrm{Hu}$ and Russell Eberhart. Solving Constrained Nonlinear Optimization Problems with Particle Swarm Optimization. In Proceedings of the 6th World Multiconference on Systemics, Cybernetics and Informatics (SCI 2002), volume 5. Orlando, USA, IIIS, July 2002.

8. Xiaohui Hu, Russell C. Eberhart, and Yuhui Shi. Engineering Optimization with Particle Swarm. In Proceedings of the 2003 IEEE Swarm Intelligence Symposium, pages 53-57. Indianapolis, Indiana, USA, IEEE Service Center, April 2003.

9. J. Kennedy and R. Mendes. Population structure and particle swarm performance. In Proceedings of the 2002 IEEE Congress on Evolutionary Computation, volume 2, pages 1671-1676, Honolulu, Hawaii, USA, May 2002. IEEE Press.

10. James Kennedy and Russell C. Eberhart. Swarm Intelligence. Morgan Kauffmann Publishers, San Francisco, California, 2001.

11. Slawomir Koziel and Zbigniew Michalewicz. Evolutionary Algorithms, Homomorphous Mappings, and Constrained Parameter Optimization. Evolutionary Computation, 7(1):19-44, 1999. 
12. Kalmanje Krishnakumar. Micro-genetic algorithms for stationary and nonstationary function optimization. SPIE Proceedings: Intelligent Control and Adaptive Systems, 1196:289-296, 1989.

13. J.J. Liang, T.P. Runarsson, E. Mezura-Montes, M. Clerc, P.N. Suganthan, C.A. Coello Coello, and K. Deb. Problem definitions and evaluation criteria for the cec 2006 special session on constrained real-parameter optimization. Technical report, Nanyang Technological University, Singapore, 2006.

14. Efrén Mezura-Montes and Carlos A. Coello Coello. A simple multimembered evolution strategy to solve constrained optimization problems. Transactions on Evolutionary Computation, 9(1):1-17, February 2005.

15. Z. Michalewicz. Genetic Algorithms + Data Structures = Evolution Programs. Springer-Verlag, 1996.

16. Zbigniew Michalewicz and Marc Schoenauer. Evolutionary Algorithms for Constrained Parameter Optimization Problems. Evolutionary Computation, 4(1):1-32, 1996.

17. Ulrich Paquet and Andries p Engelbrecht. A New Particle Swarm Optimiser for Linearly Constrained Optimization. In Proceedings of the Congress on Evolutionary Computation 2003 (CEC'2003), volume 1, pages 227-233, Piscataway, New Jersey, December 2003. Canberra, Australia, IEEE Service Center.

18. T.P. Runanarsson and X. Yao. Stochastic ranking for constrained evolutionary optimization. IEEE Transactions on Evolutionary Computation, 4(3):248-249, September 2000.

19. Y. Shi and R.C. Eberhart. A modified particle swarm optimizer. In Proceedings of the 1998 IEEE Congress on Evolutionary Computation, pages 69-73. IEEE Press, 1998.

20. Gregorio Toscano Pulido and Carlos A. Coello Coello. A constraint-handling mechanism for particle swarm optimization. In Proceedings of the 2004 IEEE Congress on Evolutionary Computation (CEC'2004), volume 2, pages 1396-1403, Portland, Oregon, USA, June 2004. IEEE Press. 